\title{
AERIAL IMAGES AND LIDAR DATA FUSION FOR DISASTER CHANGE DETECTION
}

\author{
J.C. Trinder ${ }^{\mathrm{a}}$, M. Salah ${ }^{\mathrm{b}, *}$ \\ ${ }^{a}$ School of Surveying and Spatial Information Systems, The University of New South Wales, \\ UNSW SYDNEY NSW 2052, Australia - (j.trinder@unsw.edu.au) \\ ${ }^{\mathrm{b}}$ Dept. of Surveying, Faculty of Engineering Shoubra, Benha University, 108 Shoubra Street, Cairo, Egypt - \\ (engmod2000@yahoo.com)
}

KEYWORDS: LiDAR, Aerial Images, Change detection, Building extraction, Feature extraction.

\begin{abstract}
:
Potential applications of airborne LiDAR for disaster monitoring include flood prediction and assessment, monitoring of the growth of volcanoes and assistance in the prediction of eruptions, assessment of crustal elevation changes due to earthquakes, and monitoring of structural damage after earthquakes. Change detection in buildings is an important task in the context of disaster monitoring, especially after earthquakes. Traditionally, change detection is usually done by using multi-temporal images through spectral analyses. This provides two-dimensional spectral information without including heights. This paper will describe the capability of aerial images and LiDAR data fusion for rapid change detection in elevations, and methods of assessment of damage in made-made structures. In order to detect and evaluate changes in buildings, LiDAR-derived DEMs and aerial images from two epochs were used, showing changes in urban buildings due to construction and demolition. The proposed modelling scheme comprises three steps, namely, data pre-processing, change detection, and validation. In the first step for data pre-processing, data registration was carried out based on the multi-source data. In the second step, changes were detected by combining change detection techniques such as image differencing (ID), principal components analysis (PCA), minimum noise fraction (MNF) and postclassification comparison (P-C) based on support vector machines (SVM), each of which performs differently, based on simple majority vote. In the third step and to meet the objectives, the detected changes were compared against reference data that was generated manually. The comparison is based on two criteria: overall accuracy; and commission and omission errors. The results showed that the average detection accuracies were: $78.9 \%, 81.4 \%, 82.7 \%$ and $82.8 \%$ for post-classification, image differencing, PCA and MNF respectively. On the other hand, the commission and omission errors of the results improved when the techniques were combined compared to the best single change detection method. The proposed combination of techniques gives a high accuracy of $92.2 \%$ for detection of changes in buildings. The results show that using LiDAR data in the detection process improves the accuracy of feature detection by $14.9 \%$ compared with using aerial photography alone.
\end{abstract}

\section{INTRODUCTION}

An up-to-date building database is a crucial requirement for reliable disaster damage assessment. Change detection employing LiDAR (Light Detection and Ranging) data is a useful tool for damage detection, particularly for collapsed multi-floored buildings (Tuong et al., 2004). LiDAR systems are active acquisition systems equipped with a laser scanner, a Global Positioning System (GPS) receiver and an Inertial Navigation System (INS). They emit infrared laser pulses at high frequency and record the time of flight of the return pulses. By combining the LiDAR distance with GPS and INS data the $\mathrm{X}, \mathrm{Y}$ and $\mathrm{Z}$ coordinates of ground points can be determined. The intensity of the returns can also be recorded. There are basically two types of LiDAR systems: discrete and waveform. A short pulse $(\sim \mathrm{ns})$ is emitted from the laser and in discrete systems one or more discrete distances and intensities are recorded. Waveform systems record the full waveform of the return signal. This paper will limit its discussion to data derived from discrete lidar systems.

Methods of change detection can mainly be divided into two categories:

- The determination of the difference of classifications of a surface obtained at two periods;

- The direct determination of change between two data sets.
Detecting changes by supervised classification is unreliable when the appearances of non-buildings and buildings are similar. Furthermore, using spectral information to detect change does not consider the situation when the differences occur in shape instead of colour (Huang and Chen, 2007). A number of research results, such as Knudsen and Olsen (2003), Matikainen et al. (2004), Walter (2004a,b) and Nielsen and Canty (2011) belong to the first category above. The second category [Murakami et al., 1999; Jung, 2004] is unable to determine the land category because no classification is used. It is also observed that trees often cause mistakes in the output of research.

Even though aerial photography has been conventionally employed for change detection [Niederöst, 2001; Knudsen and Olsen, 2003; Walter, 2004a; Walter, 2004b], it is subject to several unavoidable problems such as: shadows in the scenes acquired over dense urban areas with many skyscrapers; the spectral information of certain features in aerial photography is diverse and ill-defined (Knudsen and Olsen, 2003); and perspective projection causes relief displacement of buildings, which requires height information to correct. Therefore, the employment of LiDAR data rather than spectral information derived from aerial photographs offers important advantages (Tuong, et al., 2004). It allows obtaining 3D point clouds of the surface with high density as well as high accuracy. Moreover,

\footnotetext{
* Corresponding author.
} 
the method is capable of collecting data over large areas in a short time (Baltsavias, 1999).

Instead of the multi-spectral imagery that was often used in the past, many change detection methods using LiDAR data have been proposed. Murakami et al., (1999) carried out change detection of buildings using LiDAR data in Japan. That study was a simple comparison between two datasets. Tuong et al., (2004) presented an automatic method for LiDAR-based change detection of buildings in dense urban areas. Walter (2004b) used LiDAR data in an object-based classification to determine the land-use category after the observation of land phenomena. Matikainen et al. (2004) divided a LiDAR point cloud into homogeneous areas, and then extracted information to discover the building areas for change detection. Girardeau-Montau et al. (2005) directly used point-to-point position relations for change detection. Brzank et al., (2009) presented a new method to detect and evaluate morphologic changes of the Wadden Sea based on the extraction of structure lines of tidal channels from LiDAR data.

It is worth mentioning that, as change detection is an important step in data updating, some researchers used spectral-based methods such as iterative principal components analysis (IPCA) to determine temporal distance in feature space and combine it with a Bayesian decision rule to determine the presence of change (Spitzer et al., 2001). Clifton (2003) describes training neural networks to learn expected changes between images and to then identify pixel changes which do not match what is "expected". Hashimoto et al. (2011) proposed a knowledgebased change detection approach, which can obtain change information that includes not only land cover changes, but also contextual changes, such as types of damage caused by natural hazards. This approach mainly consists of two processes: information extraction and change inference using a Bayesian network. Information extraction employs object-based image analysis for extracting spatial information. Change inference uses extracted information and the Bayesian network constructed from knowledge of the change detection process. To demonstrate this approach, change detection of mudslide damage caused by heavy rain in Yamaguchi Pref., Japan was conducted. Some other methods used multi-temporal highresolution imagery to detect changes in spectral difference or used supervised classification to determine building positions for comparisons of two epochs for change detection (Knudsen and Olsen, 2003; Kumar, 2011).

Research on change detection by aerial images and LiDAR data fusion has been undertaken so that the strengths of each data type can compensate for the weaknesses of the other. Low contrast, occlusions and shadow effects in the images can be compensated by the accurately defined planes in the LiDAR data (Sohn and Dowman, 2003). On the other hand, the poorly defined edges in the LiDAR data can be compensated by the accurately defined edges in the aerial images.

Huang and Chen (2007) included LiDAR data and aerial images to detect the changes of building models. Chien and Lin (2010) developed a new method to find changes within 3D building models in the region of interest with the aid of LiDAR data. Their modelling scheme comprises three steps, namely, data pre-processing, change detection in building areas, and validation. Research findings clearly indicate that a doublethreshold strategy improves the overall accuracy from $93.1 \%$ to 95.9\%.
To evaluate the contribution of the images and LiDAR data in the change detection process in this study, four change detection methods were each tested using images and LiDAR data separately and in combination, to determine the accuracy of change detection against a reference map.

The paper is organised as follows. Section 2 describes the study areas and data sources. Section 3 describes the experiments while Section 4 presents and evaluates the results. We summarise our results in Section 5.

\section{STUDY AREAS AND DATA SOURCES}

\subsection{Aerial images and LiDAR data}

In order to demonstrate the capability of the proposed change detection method, two aerial images and LiDAR datasets were available, acquired on different occasions over Coffs Harbour in NSW Australia. The characteristics of aerial images and LiDAR datasets are summarized in Table 1. Data was provided over a dense urban area which includes residential buildings, large buildings, a network of main and local roads, open and green areas as well as trees as shown in figure 1 .

Table 1: Characteristics of aerial images and LiDAR datasets.

\begin{tabular}{|c|l|l|}
\hline $\begin{array}{c}\text { Earlier aerial } \\
\text { image }\end{array}$ & $\begin{array}{l}\text { Resolution } \\
\text { Acquisition time }\end{array}$ & $50 \mathrm{~cm}$ \\
\hline \multirow{2}{*}{ Later aerial image } & Resolution & $10 \mathrm{~cm}$ \\
& Acquisition time & $12 / 2009$ \\
\hline \multirow{2}{*}{ LiDAR data } & Density & $1.5 \mathrm{pts} / \mathrm{m}^{2}$ \\
& Acquisition time & $9 / 2009 \& 12 / 2009$ \\
\hline
\end{tabular}
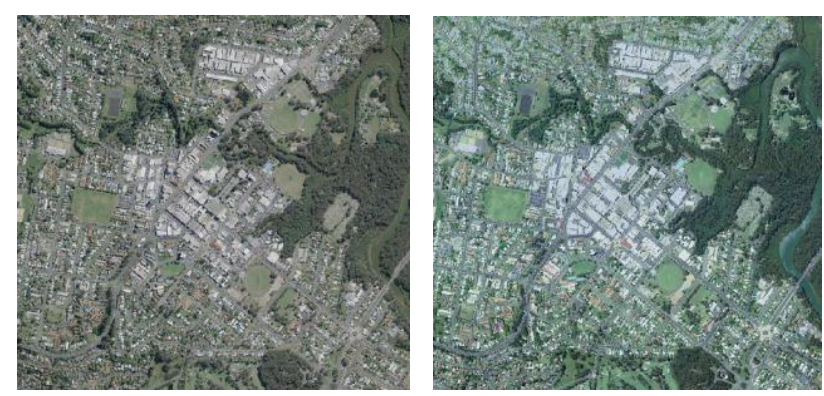

Figure 1. Earlier and later aerial images of the test site.

\subsection{Reference data}

In order to accurately evaluate the performance of the proposed change detection method, changes were visually interpreted and digitized independently of their size. The reference data is a four-class thematic image, typically divided into the four categories of: 'background'; 'decreased', 'increased'; and 'unchanged'.

\section{METHODOLOGY}

\subsection{Pre-processing}

First, both DEMs were registered to each other based by a projective transformation. The registration process resulted in small Root Mean Square (RMS) errors that did not exceed 0.15 $\mathrm{m}$ in both $\mathrm{X}$ and $\mathrm{Y}$ directions. Then, both aerial images were registered to the LiDAR DSM based on a projective transformation. The registration process resulted in small Root Mean Square Errors (RMSE) that did not exceed $1.2 \mathrm{~m}$ in both $\mathrm{X}$ and $\mathrm{Y}$ directions. Following the transformation, the images were resampled to $1 \mathrm{~m}$ pixel size. A grid format is preferred to 
the raw point cloud format to speed up the processing, particularly when there is a direct comparison of the two datasets. In order to obtain high image quality and to reduce the processing time, a bilinear interpolation was applied for the resampling process. The bilinear interpolation can result in a better quality image than nearest neighbourhood resampling and requires less processing than cubic convolution.

\subsection{Main-processing}

Four different change analyses were performed to evaluate the efficacy of aerial images and LiDAR data for detecting changes occurring in two epochs. The four methods include: image differencing; principal components analysis (PCA); minimum noise fraction (MNF); and Post-Classification based on support vector machine (SVM). After these steps, a simple majority vote has been applied to generate the change detection image. All the methods proposed in this research were implemented through programs generated by the authors in a Matlab environment. An interface was developed to enable the user to: detect changes through the aforementioned four methods; combine votes derived from all methods; generate a change detection image; and evaluate the change detection results. The workflow for this investigation is shown in figure 2 .

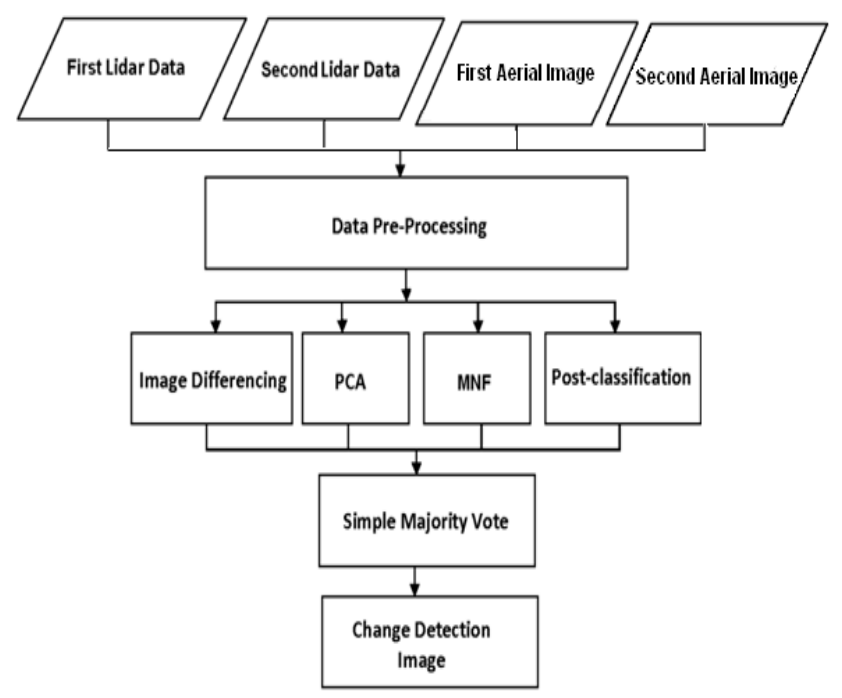

Figure 2. The workflow for change detection analysis.

In the first change detection technique, differences that exceed a user-specified threshold of 10 pixels in area and $0.30 \mathrm{~m}$ in height, double the LiDAR system accuracy, were computed and highlighted. In the image differencing method, the second image is subtracted from the first image to provide the difference and highlight changes. The second image is more recent and the differences reflect changes over time. After application of image differencing, increases in height values that are more than the predefined thresholds, are highlighted as increases, while decreases in height values that are more than the predefined thresholds, are highlighted as decreases. The result is a grey scale image composed of a single band of continuous data that reflects the changes. The change image is a four-class thematic image, typically divided into the four categories of: background; decreased, increased; and unchanged. Although the calculation is simple, the interpretation requires knowledge about the area, because every difference relates to a certain location but not necessarily to the same object.

In the second change detection technique, principal components analysis (PCA) has been applied to detect changes. Principal components analysis (PCA) is commonly applied for orthogonal data transformations by maximizing the spectral variability, by decreasing the redundancy of information contained in multiple spectral bands (Armenakis et al., 2003). PCA components are based on statistical relationships that are difficult to interpret, and are variable between different landscapes and different dates for a single landscape (Collins and Woodcock 1994). PCA is a linear transformation of the data along perpendicular axes of maximum variance between data sets (Legendre and Legendre 1998). The first eigenvector sorts pixels along an axis of highest correlation between data sets. Pixels on this axis have not significantly changed between the two images. The second eigenvector is perpendicular to the first, and therefore sorts pixels that represent differences between data sets.

The third change detection technique was based on the minimum noise fraction Transform (MNF) as modified from Green et al. (1988). MNF is a linear transformation that consists of the following separate principal components analysis rotations: (i) The first rotation uses the principal components of the noise covariance matrix to decorrelate and rescale the noise in the data (a process known as noise whitening), resulting in transformed data in which the noise has unit variance and no band-to-band correlations; (ii) The second rotation uses the principal components derived from the original image data after they have been noise-whitened by the first rotation and rescaled by the noise standard deviation. The inherent dimensionality of the data is determined by examining the final eigenvalues and the associated images. For the best results, and to save disk space, only those bands with high eigenvalues have been output. Images with eigenvalues close to 1 are mostly noise.

In the fourth change detection technique, post-classification comparison was performed in order to detect changes. The data were classified using a support vector machine classifier (SVM), then the classification results were compared and the differences were extracted. The objective is to classify the input data into four primary classes of interest, namely buildings, trees, roads, and ground. SVMs are based on the principles of statistical learning theory (Vapnik, 1979) and delineate two classes by fitting an optimal separating hyperplane (OSH) to those training samples that describe the edges of the class distribution. As a consequence they generalize well and often outperform other algorithms in terms of classification accuracies. Furthermore, the misclassification errors are minimized by maximizing the margin between the data points and the decision boundary. Since the One-Against-One (1A1) technique usually results in a larger number of binary SVMs and then in subsequently intensive computations, the OneAgainst-All (1AA) technique was used to solve for the binary classification problem that exists with the SVMs and to handle the multi-class problems. The Gaussian radial basis function (RBF) kernel has been used, since it has proved to be effective with reasonable processing times in remote sensing applications.

Then a simple majority vote, which can be more effective than more complex voting strategies (Waske, 2007), was used to generate the final result. If change detection algorithm $\mathrm{c}_{\mathrm{i}}$ assigns a given pixel to class label $\omega_{\mathrm{j}}$, then we say that a vote is given to $\omega_{\mathrm{i}}$. After counting the votes given to each class label by all 
detection algorithms, the class label that receives the highest number of votes is taken as the final output. It is worth mentioning that all votes are of equal weight and independent of height differences. When the four detection methods give completely different decision for a given pixel, which does not convey any information, the decision from the method with highest overall detection accuracy is considered.

As a last step, the smaller detected regions were merged into larger neighbouring homogeneous ones or deleted according to an arbitrary $1 \mathrm{~m}$ distance and $30 \mathrm{~m}^{2}$ area thresholds respectively. The area threshold represents the expected minimum change area, while the distance threshold was set to 1 $\mathrm{m}$ to fill in any gaps within the detected region. Regions were retained if they were larger than the given area threshold and/or were adjacent to a larger homogeneous region by a distance less than $1 \mathrm{~m}$.

Finally, region borders were cleaned by removing structures that were smaller than 5 pixels and that were connected to the region border. There was a compromise between cleaning thresholds less than 5 pixels, which may leave the original buildings uncleaned, and thresholds greater than 5 pixels which may remove parts of the detected region. The result was an image that represents the detected changes without noisy features and also without holes. Because ground and vegetation usually result in wrong detection, we removed ground and vegetation areas in LiDAR data and aerial images.

\subsection{Evaluation of the change detection results}

In order to evaluate the performance of the adopted method for change detection from aerial images and LiDAR data, the results have been checked based on two different methods:

(i) The overall detection accuracy which was assessed using the reference data based on equation 1 :

$$
O D A=\frac{N C P}{N R P}
$$

Where $O D A$ is the overall detection accuracy; $N C P$ is the total number of correctly detected pixels and $N R P$ is the total number of reference pixels.

(ii) The omission and commission errors: since the overall detection accuracy is a global measure the performance of the proposed combination method was also evaluated by determining the commission and omission errors (Congalton, 1991). Unlike overall detection accuracy, commission and omission errors clearly show how the performance of the proposed methods improve the results or causes a deterioration of results for each individual class compared to the reference data.

$$
\begin{aligned}
& C E_{I}=\frac{A_{1}+A_{2}+A_{3}}{R_{1}} \\
& O E_{I}=\frac{B_{1}+B_{2}+B_{3}}{R_{1}}
\end{aligned}
$$

$C E_{I}$ and $O E_{I}$ are commission and omission errors of class increased; $A_{1}, A_{2}$ and $A_{3}$ are the numbers of incorrectly identified pixels of class increased associated with classes decreased, background and unchanged; $R_{l}$ is the total number of pixels of the class increased as observed in the reference data; $B_{1}, B_{2}$ and $B_{3}$ are the numbers of unrecognized pixels that should have identified as belonging to the class increased. The same is applicable for the class decreased.

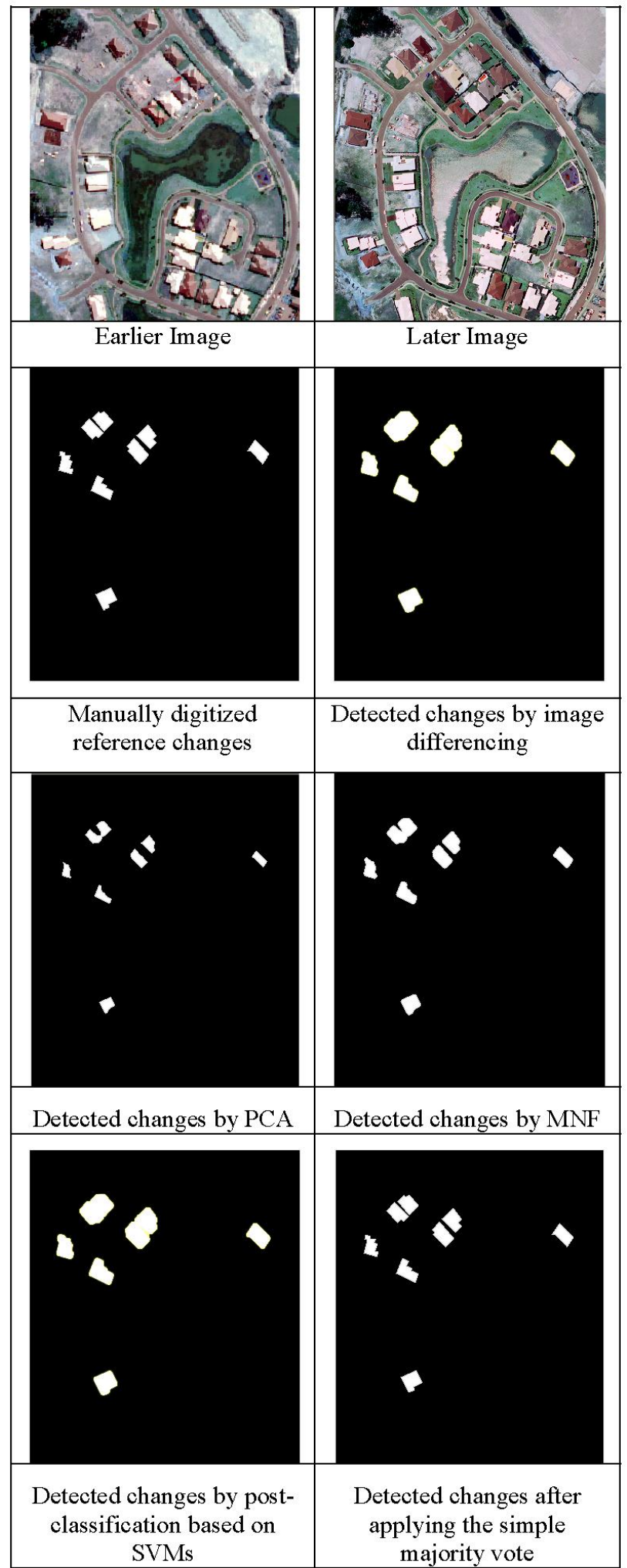

Figure 3. A typical example showing the results in a sub-area of the whole test area. 


\section{RESULTS AND ANALYSIS}

Figure 3 is a typical example showing the results in a sub-area of the whole test area. For the detected changes, the white colour indicates a change while black colour refers to both background and unchanged. It can clearly be seen that the detected changes for PCA are eroded as compared to the reference data. This trend can also be observed for postclassification results. On the other hand, the detected changes for image differencing are larger. However, the erosion effect is less when the MNF and the simple majority vote combination are applied.

The overall accuracies of individual change detection techniques, based on the reference data, are given in Table 2. MNF performed the best with $82.8 \%$ detection accuracy, followed by PCA, image differencing and post-classification with detection accuracies of $82.7 \%, 81.4 \%$ and $78.9 \%$ respectively. It is clear that the combination of the 4 change detection methods based on simple majority vote resulted in higher accuracy of change detection than when each detection method was used individually. An improvement in detection accuracy of $9.4 \%$ was obtained from the simple majority voting combination algorithm compared with the best individual detection method, MNF.

Table 3 shows the commission and omission errors based on the proposed method in cases of: aerial images; LiDAR data; and aerial images plus LiDAR data. It can be seen that a considerable amount of the misclassified pixels have been recovered by the combined processing of aerial images and LiDAR data. As well, omission and commission errors are comparable for aerial images, LiDAR data and when fused, as shown in table 3 . The table also indicates the capabilities of the method of combining the three methods to detect changes from aerial images and LiDAR data.

Table 2: Overall detection accuracy for single detection techniques compared with results when they are used in combination (SMV).

\begin{tabular}{|c|c|c|c|}
\hline \multirow{2}{*}{$\begin{array}{c}\text { Metho } \\
\text { d }\end{array}$} & \multicolumn{3}{|c|}{ Detection accuracy } \\
\cline { 2 - 4 } & Aerial Images & LiDAR Data & $\begin{array}{c}\text { Aerial Images \& } \\
\text { LiDAR Data }\end{array}$ \\
\hline ID & 62.90 & 72.36 & 81.40 \\
PCA & 64.16 & 73.85 & 82.73 \\
MNF & 64.58 & 73.75 & 82.82 \\
P-C & 66.50 & 69.42 & 78.87 \\
SMV & 77.35 & 81.61 & 92.23 \\
\hline
\end{tabular}

Table 3: Change detection errors of the algorithm for combining the three change detection methods for aerial images, LiDAR data and when these data are fused. Com. and Om. Stand for commission and omission errors respectively.

\begin{tabular}{|c|c|c|c|c|c|}
\hline \multicolumn{2}{|c|}{ Aerial Images } & \multicolumn{2}{c|}{ LiDAR Data } & \multicolumn{2}{c|}{$\begin{array}{c}\text { Aerial Images \& } \\
\text { LiDAR Data }\end{array}$} \\
\hline Om. & Com. & Om. & Com. & Om. & Com. \\
$(\%)$ & $(\%)$ & $(\%)$ & $(\%)$ & $(\%)$ & $(\%)$ \\
20.3 & 10.8 & 11.4 & 13.1 & 9.0 & 8.9 \\
\hline
\end{tabular}

\section{CONCLUSION}

In this paper, we have applied a powerful method to combine change detection techniques with different performances based on simple majority vote. To test the algorithm, four change detection methods were based on aerial images and LiDAR data of different two epochs. The results showed an improvement in terms of detection accuracy as well as omission and commission errors. Detection accuracies of individual algorithms were $78.9 \%, 81.4 \%, 82.7 \%$ and $82.8 \%$ for post-classification, image differencing, PCA and MNF respectively, whereas the proposed combination algorithm gave an accuracy of $92.2 \%$ which is an improvement of around $9.4 \%$. On the other hand, the proposed method showed a high level of automation in the change detection process. These results demonstrate the overall advantages of the proposed algorithm for change detection that could be applicable for detecting changes in buildings damaged in a disaster such as an earthquake. If two LiDAR flights could be carried out before and after an earthquake, the change detection results can reveal the collapsed buildings. It would be a positive approach to use discrete LiDAR point clouds instead of DSM images to alleviate interpolation errors.

\section{REFERENCES}

Armenakis C., F. Leduc, I. Cyr, F. Savopol, F. Cavayas (2003). A comparative analysis of scanned maps and imagery for mapping applications. ISPRS Journal of Photogrammetry and Remote Sensing, 57:304-14.

Baltsavias E. (1999). Airborne laser scanning: basic relations and formulas. ISPRS Journal of Photogrammetry and Remote Sensing, 54(2-3), pp. 199-214.

Chen L. C. (2010). Detection of building changes from aerial images and light detection and ranging (LIDAR) data. Journal of Applied Remote Sensing, Vol. 4, 041870 (19 November 2010).

Clifton C. (2003). Change Detection in Overhead Imagery using Neural Networks. International Journal of Applied Intelligence, 18(2):215-234.

Collins J. B., C. E. Woodcock (1994). Change detection using the Gramm-Schmidt transformation applied to mapping forest mortality. Remote Sensing of Environment, 50:267-79.

Congalton R.G. (1991). A review of assessing the accuracy of classifications of remotely sensed data. Remote Sensing of Environment, 37(1):35-46.

Girardeau-Montaut D., Roux M., Marc R., Thibault G. (2005). Change Detection on Points Cloud Data Acquired with a Ground Laser Scanner, International Archives of Photogrammetry, Remote Sensing and Spatial Information Sciences, XXXVI (Pt. 3/W19):30-35.

Green A. A., Berman M., Switzer P., Craig M. D. (1988). A transformation for ordering multispectral data in terms of image quality with implications for noise removal: IEEE Transactions on Geoscience and Remote Sensing, v. 26, no. 1, p. 65-74.

Hashimoto S., Tadono T., Onosatoa M., Horib M. and Moriyamab T. (2011). An Approach for Automatic Change Inference in High Resolution Satellite Images. 34th International Symposium on Remote Sensing of Environment, The GEOSS Era: Towards Operational Environmental Monitoring, Sydney, Australia, 10-15 April 2011.

Heipke C., Mayer H., Wiedemann C., Jamet O. (1997). 
Evaluation of automatic road extraction. International Archives of Photogrammetry and Remote Sensing, XXXII-2-3W3, Haifa, Israel, pp. 47-56.

Huang C., Chen L. (2007). Detection of Building Changes from LiDAR Data and Aerial Imagery. In: ACRS 2007: proceedings of the 28th Asian conference on remote sensing, 12-16 November 2007, Kuala Lumpur, Malaysia.

Jung F. (2004). Detecting Building Changes from Multitemporal Aerial Stereopairs. ISPRS Journal of Photogrammetry and Remote Sensing, 58:187-201.

Knudsen T., Olsen B. P. (2003). Automated Change Detection for Updates of Digital Map Databases, Photogrammetric Engineering and Remote Sensing, vol. 69 (11), pp. 1289-1297, 2003.

Kumar A. (2011). Temporal Changes in Mangrove Cover Between 1972 and 2001 Along the South Coast of the Arabian Gulf. 34th International Symposium on Remote Sensing of Environment, The GEOSS Era: Towards Operational Environmental Monitoring, Sydney, Australia, 10-15 April 2011.

Legendre P., L. Legendre (1998). Numerical Ecology. Elsevier Science, Amsterdam. 853 p.

Li Y. and Wu H., 2008. Adaptive Building Edge Detection by Combining LiDAR Data and Aeria Images. The International Archives of the Photogrammetry, Remote Sensing and Spatial Information Sciences. Vol. XXXVII. Part B1. Beijing 2008

Matikainen L., Hyyppä J., Hyyppä H. (2004). Automatic Detection of Changes from Laser Scanner and Aerial Image Data for Updating Building Maps. International Archives of Photogrammetry, Remote sensing and Spatial Information Sciences, XXXV (B2):434-439.

Murakami H., Nakagawa K., Hasegawa H. Shibata T. (1999). Change Detection of Buildings Using an Airborne Laser Scanner, ISPRS Journal of Photogrammetry and Remote Sensing, 54:148-152.

Niederöst M. (2001). Automated update of building information in maps using medium-scale imagery $(1: 15,000)$, Automatic Extraction of Man-Made Objects from Aerial and Space Images (III), Baltsavias, E., Gruen, A., Van Gool, L. (Eds.), Balkema, Lisse, pp. 161-170.

Nielsen A. A., Canty M. J. (2011). A Method for Unsupervised Change Detection and Automatic Radiometric Normalization in Multispectral Data. 34th International Symposium on Remote Sensing of Environment, The GEOSS Era: Towards Operational Environmental Monitoring, Sydney, Australia, 10-15 April 2011.

Sohn, G. and Dowman, I., 2003, Building extraction using LiDAR DEMs and ikonos images. In Proceedings of the ISPRS working group III/3 workshop ' $3-D$ reconstruction from airborne laserscanner and InSAR data', XXXIV-3/W13, 8-10 October 2003, Dresden, Germany.

Spitzer H., R. Franck, M. Kollewe, N. Rega, A. Rothkirch, R. Wiemker (2001). Change Detection with $1 \mathrm{~m}$ Resolution Satellite and Aerial Images. In: Proceedings of the IEEE 2001 International Geoscience and Remote Sensing Symposium. Vol. 5, pp. 2256-2258.

Clifton C. (2003). Change Detection in Overhead Imagery using Neural Networks. International Journal of Applied Intelligence,

\section{$18(2): 215-234$}

Tuong T.V., Matsuoka M., Yamazaki F. (2004). LiDAR-based Change Detection of Buildings in Dense Urban Areas, Proceedings of the IEEE International Geoscience and Remote Sensing Symposium, Vol. 5, pp.3413-3416.

Vapnik V. (1979). Estimation of Dependences Based on Empirical Data [in Russian]. Nauka, Moscow, 1979. (English translation: Springer Verlag, New York, 1982).

Walter V. (2004a). Object-based classification of remote sensing data for change detection, ISPRS Journal of Photogrammetry \& Remote Sensing, vol. 58, pp. 225- 238, 2004.

Walter V. (2004b). Object-based Evaluation of LiDAR and Multispectral Data for Automatic Change Detection in GIS Databases, IAPRS, 35(B2):723-728.

Waske B. (2007). Classifying multisensor remote sensing data: concepts, algorithms and applications. PhD thesis, Bonn University, Germany.

\section{ACKNOWLEDGEMENTS}

The authors wish to thank NSW Department of Land and Property Information for the LiDAR and image data. 